\title{
Resveratrol Improves Cognitive Impairment by Regulating Apoptosis and Synaptic Plasticity in Streptozotocin- Induced Diabetic Rats
}

\author{
Zhiyan Tian ${ }^{\mathrm{a}}$ Jinhua Wang ${ }^{\mathrm{a}}$ Ming Xub Yan Wang ${ }^{\mathrm{a}}$ Miao Zhang ${ }^{\mathrm{a}}$ Yuying Zhou
}

aDepartment of Neurology, Tianjin Huanhu Hospital, Jinnan District, Tianjin, ${ }^{b}$ Department of Oral Pathology, College and Hospital of Stomatology, Hebei Medical University; Key Laboratory of Stomatology, Shijiazhuang, Hebei Province, PR China

\section{Key Words}

Resveratrol $\bullet$ Cognitive impairment $•$ Apoptosis $\bullet$ Synaptic plasticity $\bullet$ Diabetic rats

\begin{abstract}
Aims: To investigate the effects of resveratrol on cognitive impairment in streptozotocin (STZ)induced diabetic rats and to explore the mechanisms of that phenomenon. Methods: Sixty healthy male Sprague Dawley rats were randomly divided into four groups: normal control group (Con group, $\mathrm{n}=15$ ), Res group (normal Sprague Dawley rats treated with resveratrol, $n=15$ ), diabetes mellitus group (DM group, $n=15$ ) and $D M+$ Res group (diabetic rats treat with resveratrol, $n=15$ ). Streptozotocin (STZ) was injected intraperitoneally to establish the diabetic model. One week after diabetic model induction, the animals in the Res group and the $\mathrm{DM}+$ Res group received resveratrol intraperitoneally once a day for consecutive 4 weeks. The Morris water maze test was applied to assess the effect of resveratrol on learning and memory. To explore the mechanisms of resveratrol on cognition, we detected the protein expression levels of Caspase-3, Bcl-2, Bax, NMDAR1 (N-Methyl-d-Aspartate receptor) and BDNF (Brain Derived Neurotrophic Factor) via western blotting analysis. Results: Resveratrol has no obvious effect on normal SD rats. Compared to Con group, cognitive ability was significantly impaired with increased expression of Caspase-3, Bax and down-regulation of $\mathrm{BCl}-2$, NMDAR1 and BDNF in diabetic rats. By contrast, resveratrol treatment improved the cognitive decline. Evidently, resveratrol treatment reversed diabetes-induced changes of protein expression. Conclusions: Resveratrol significantly ameliorates cognitive decline in STZ-induced diabetic model rats. The potential mechanism underlying the protective effect could be attributed to the inhibition of hippocampal apoptosis through the $\mathrm{Bcl}-2$, Bax and Caspase-3 signaling pathways and improvement of synaptic dysfunction. BDNF may also play an indispensable role in this mechanism.




\section{Introduction}

Diabetes mellitus, one of the most severe metabolic disorders in humans globally, is characterized by insulin resistance and impaired insulin secretion [1]. Diabetes causes numerous complications, such as retinopathy, renal failure, cardiovascular disease and autonomic neuropathy [2]. Evidence suggests that diabetes also affects the central nervous system, including the hippocampus, a part of the limbic system, which is associated with cognitive functions [3]. Several studies [4, 5] have revealed the tight association of diabetes with cognitive impairment and dementia. It is estimated that 382 million adults had diabetes in 2013, and this number is predicted to reach 592 million in 2035 [6]. The increasing incidence of diabetes causes a serious socioeconomic burden. Nevertheless, the precise mechanisms underlying diabetes-related cognitive deficits are still elusive. Therefore, the mechanisms of and effective treatment for cognitive impairment in diabetes mellitus are urgently needed.

Resveratrol is a natural phenolic compound enriched in the skin of red grapes and red wine and also found abundantly in Polygonum cuspidatum. Several studies have demonstrated that resveratrol has a variety of biological and pharmacological effects, including cardioprotective, antioxidant, anti-inflammatory, and antiapoptotic activities [712]. Some studies have used resveratrol to reverse cognitive deficits in alcohol- and diabetesinduced cognitive deficits in rats $[13,14]$. However, the potential neuroprotective effects of resveratrol against cognitive decline and the mechanisms remain unknown. The purpose of our research was to explore whether resveratrol could prevent memory deficit in STZinduced diabetic rats and, if so, what its possible mechanisms are.

\section{Materials and Methods}

\section{Animals}

Sixty healthy male Sprague Dawley rats (2 months old, 180-210 g) were obtained from the Animal Center of Tianjin. All of the experiments were performed in compliance with the regulations approved by the ethics committee of Tianjin Huanhu Hospital. Rats were housed in a room, at $21-25^{\circ} \mathrm{C}$ in a 12 -hour lightdark cycle. All rats had access to food and water ad libitum. All efforts were made to reduce the number of animals used and their suffering.

\section{Model induction and drug administration}

Animals were randomly divided into four groups: normal control group (Con group, $n=15$ ), Res group (normal Sprague Dawley rats treat with resveratrol, $n=15$ ), diabetes mellitus group (DM group, $n=15$ ) and $\mathrm{DM}+$ Res group (diabetic rats treat with resveratrol, $\mathrm{n}=15$ ). Thirty rats (DM group and DM + Res group) received STZ (abcam, $60 \mathrm{mg} / \mathrm{kg}$, dissolved in normal saline) by intraperitoneal injection. Diabetic models were considered to be successful if blood glucose levels were above $16.7 \mathrm{mM}$ at 72 hours after STZ injection (blood samples were taken from the tail vein to measure glucose levels). 15 rats without STZ injection were used as a normal control. One week after diabetic model induction, the animals in Res group and DM + Res group received resveratrol (Sigma, $80 \mathrm{mg} / \mathrm{kg}, 10 \mathrm{ml} / \mathrm{kg}$ ) intraperitoneally once a day for consecutive 4 weeks, while the animals from other groups received saline intraperitoneally.

Morris water maze

On the first day following the last treatment, all rats were subjected to the Morris water maze (MWM) test to assess cognitive ability. Tests were carried out at a fixed period of time every day for 5 consecutive days. A black cylindrical tank $(120 \mathrm{~cm}$ in diameter $)$ was filled with water $\left(20-24^{\circ} \mathrm{C}\right)$, made opaque with a nontoxic acrylic black color. The tank was divided into four quadrants and a circular escape platform 10 $\mathrm{cm}$ in diameter was placed at a fixed position in the center of the target quadrant. The platform was $2 \mathrm{~cm}$ under the water surface. A camera was placed above the center of the maze, and a tracking system was used to record the performance of rats. The time taken to escape onto the hidden platform (escape latency) and swimming speed were measured. If a rat failed to reach the platform within $120 \mathrm{~s}$, it was guided onto 
the platform (were it was kept for $20 \mathrm{~s}$ ), and the latency was recorded as $120 \mathrm{~s}$. In the probe trial (day 6), the platform was removed, and the rats were allowed to search for the missing platform for $120 \mathrm{~s}$. The frequency of platform crossings and time spent in the target quadrant were recorded.

\section{Blood glucose testing}

Blood samples were taken from the tail to measure glucose levels. At onset of the test and after behavioral studies, animals underwent fasting overnight, and blood glucose levels were measured with a portable glucometer (Roche, Switzerland).

\section{Western blot}

Hippocampi were dissected, rapidly frozen and kept at $-80^{\circ} \mathrm{C}$ for further use. Tissues were homogenized with ice-cold radioimmunoprecipitation assay lysis buffer ( $50 \mathrm{mM}$ Tris- $\mathrm{HCl}, 150 \mathrm{mM} \mathrm{NaCl}, 1 \%$ Triton X-100, 1\% sodium deoxycholate, $\mathrm{pH} 7.4$ ). Hippocampal lysates were separated by $12.5 \%$ SDS-PAGE and transferred onto polyvinylidene fluoride membranes. Membranes were blocked with $5 \%$ non-fat milk for $1 \mathrm{~h}$ at room temperature and then incubated overnight at $4^{\circ} \mathrm{C}$ with the following primary antibodies: Caspase-3 (1:1000, Abcam, USA), Bcl-2 (1:500, Abcam, USA), Bax (1:1000, Abcam, USA), NMDAR1 (1:1000, Abcam, USA), BDNF (1:2000, Abcam, USA) and $\beta$-actin (1:2,000, Abcam, USA). The membranes were incubated with the secondary antibodies for $1 \mathrm{~h}$ at room temperature. The membranes were detected using a chemiluminescent system. The bands were quantified using the Quantity one software.

\section{Statistical analysis}

Data are presented as the mean \pm SD. In the MWM test, escape latency was analyzed by two-way ANOVA followed by LSD post hoc test. All other data were analyzed by one-way ANOVA. Statistical analysis was performed using SPSS 13.0 software. $p<0.05$ was considered to be statistically significant.

\section{Results}

Resveratrol reversed cognitive impairments in diabetic model rats

We evaluated the learning and memory ability in different groups using the MWM experiment to determine whether resveratrol reversed cognitive impairments (Fig. 1). The escape latency to find the platform was significantly increased in DM group compared to Con group. However, the escape latency was significantly decreased after 4 weeks of resveratrol administration (Fig. 1A). In the probe test, the platform was removed. The time spent in all

Fig. 1. Results of Morris water maze test. Resveratrol enhanced learning and memory in diabetic rats. (A) Escape latency. (B) The time spent in the target quadrant after removal of the platform. (C) The number of crossings in platform area. (D) Swimming speed in different groups. ${ }^{*} p<0.05$ vs. Con group. ${ }^{\#} p<0.05$ vs. DM group.

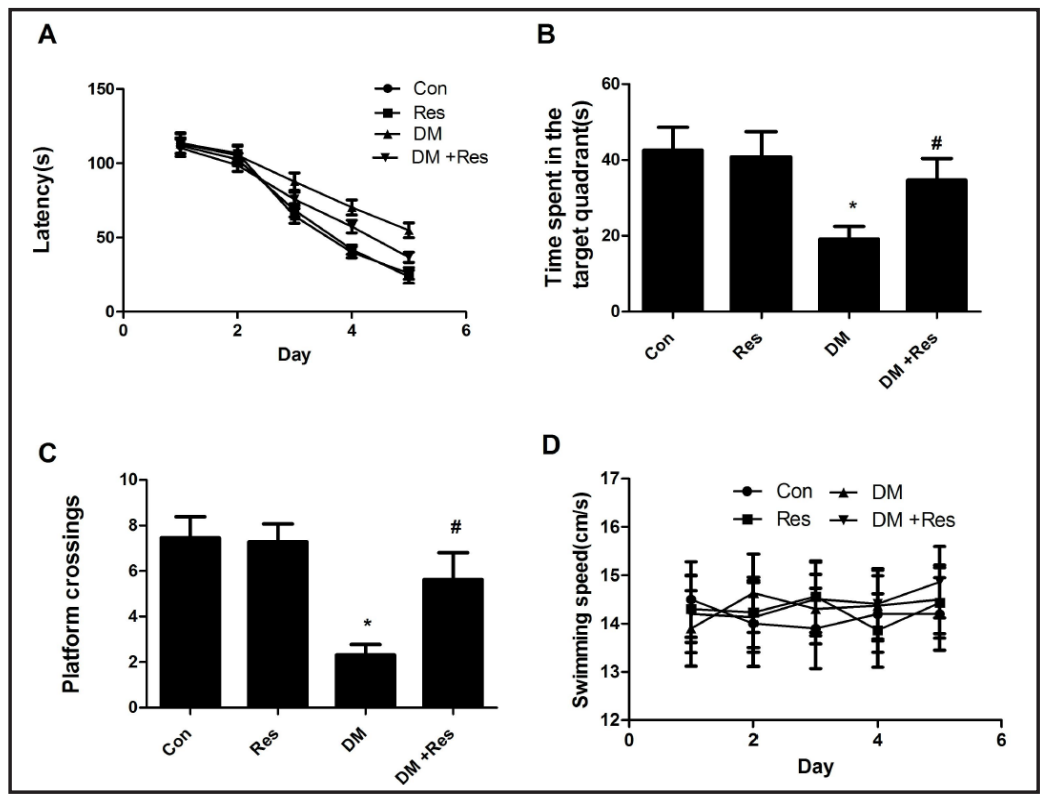


Table 1. Time spent in all quadrants. ${ }^{*} \mathrm{p}<0.05$ compared with Con group; $\# \mathrm{p}<0.05$ compared with DM group

\begin{tabular}{ccccc}
\hline Group & first quadrant & second quadrant & third quadrant & target quadrant \\
\hline Con & $31.77 \pm 6.59$ & $17.29 \pm 5.84$ & $28.13 \pm 8.19$ & $42.53 \pm 6.04$ \\
Res & $33.16 \pm 5.86$ & $19.52 \pm 4.85$ & $26.68 \pm 5.19$ & $40.76 \pm 6.67$ \\
DM & $27.58 \pm 4.73$ & $40.09 \pm 4.47$ & $32.67 \pm 8.35$ & $19.17 \pm 4.31^{*}$ \\
DM+Res & $30.12 \pm 4.81$ & $25.53 \pm 6.18$ & $29.52 \pm 5.20$ & $34.67 \pm 5.72 \#$ \\
\hline
\end{tabular}

Table 2. Effect of resveratrol on blood glucose levels (mmol/L). *p $<0.05$ compared with Con group; \#p<0.05 compared with DM group

\begin{tabular}{llc}
\hline Group & Onset of study & End of study \\
\hline Con & $6.87 \pm 2.32$ & $6.71 \pm 1.96$ \\
Res & $6.74 \pm 2.04$ & $6.57 \pm 1.75$ \\
DM & $6.69 \pm 2.22$ & $27.26 \pm 2.81^{*}$ \\
DM+Res & $6.76 \pm 1.88$ & $16.35 \pm 2.73 \#$ \\
\hline
\end{tabular}

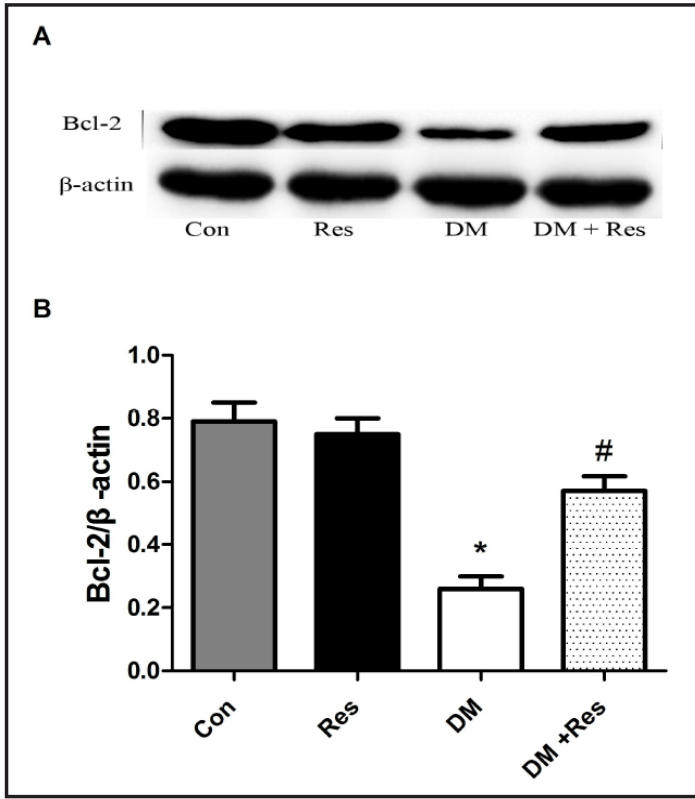

Fig. 2. Western blotting analysis reveals Bcl-2 expression. (A) Bcl- 2 and $\beta$-actin protein bands. (B) The relative protein expression of $\mathrm{Bcl}-2 .{ }^{*} p<0.05$ vs. Con group. ${ }^{*} p<0.05$ vs. DM group.

\section{A}

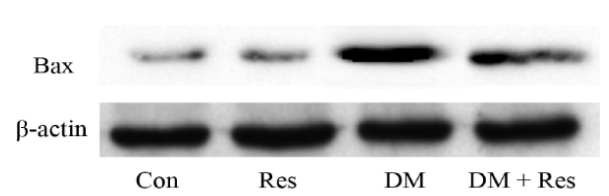

B

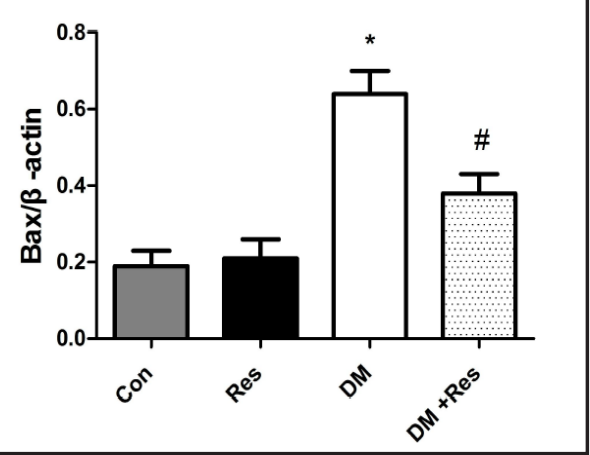

Fig. 3. Western blotting analysis reveals Bax expression. (A) Bax and $\beta$-actin protein bands. (B) The relative protein expression of $\mathrm{Bax} .{ }^{*} p<0.05$ vs. Con group. ${ }^{*} p<0.05$ vs. DM group.

quadrants is presented in Table 1. The frequency of platform crossings and time spent in the target quadrant was significantly lower in the DM group than in the Con group (Fig. 1B and Fig. 1C). By contrast, the time and frequency was significantly increased in the DM + Res group compared to the DM group. There were no significant differences in swimming speed among different groups (Fig. 1D). There were no significant differences in cognitive ability between the Con group and the Res group, indicating that resveratrol did not influence cognition in normal SD rats. 


\section{Cellular Physiology Cell Physiol Biochem 2016;40:1670-1677 \begin{tabular}{ll|l} 
and Biochemistry $\begin{array}{l}\text { DOI: 10.1159/000453216 } \\
\text { Published online: } 2016 \text { The Author(s). Published by S. Karger AG, Basel }\end{array}$ & $\begin{array}{l}\text { (c) } 2016 \text {. } \\
\text { www.karger.com/cpb }\end{array}$
\end{tabular}

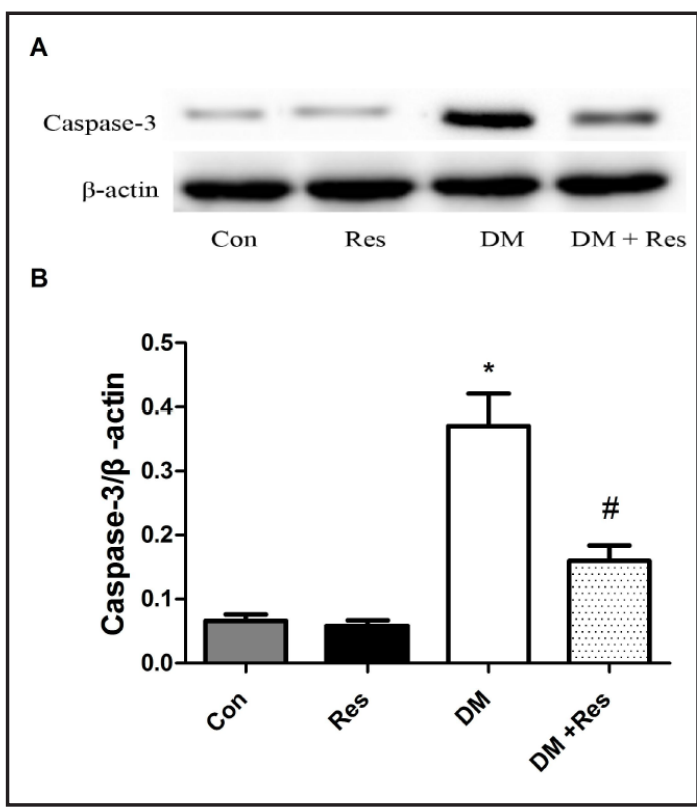

Fig. 4. Western blotting analysis reveals Caspase-3 expression. (A) Caspase- 3 and $\beta$-actin protein bands. (B) The relative protein expression of Caspase-3. ${ }^{*} p<0.05$ vs. Con group. ${ }^{*} p<0.05$ vs. DM group.

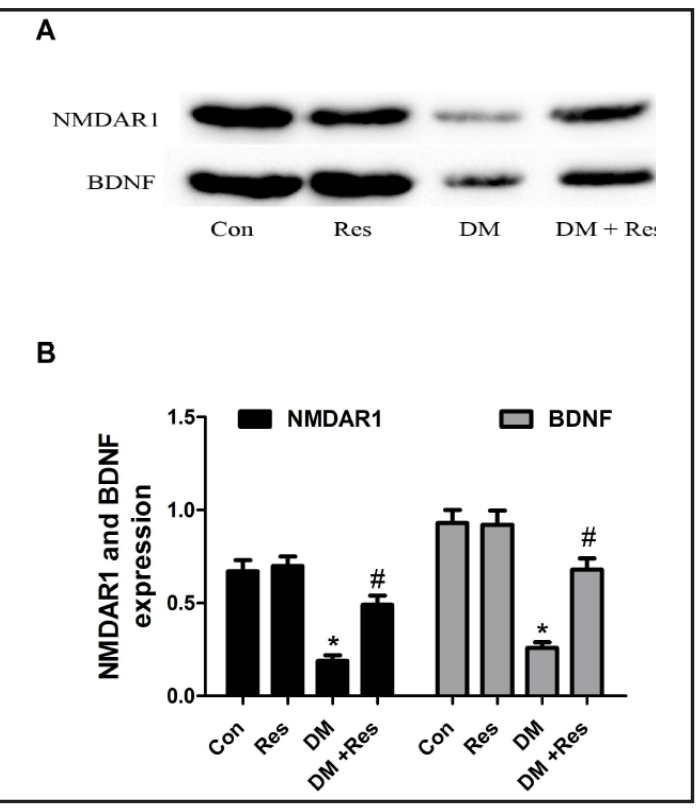

Fig. 5. Western blotting analysis reveals NMDAR1 and BDNF expression. (A) NMDAR1, BDNF protein bands. (B) The relative protein expression of NMDAR1 and BDNF. ${ }^{*} p<0.05$ vs. Con group. ${ }^{*} p<0.05$ vs. DM group.

Resveratrol decreased the blood glucose in diabetic model rats

The blood glucose levels at the onset of the experiment showed no significant differences among the groups. The blood glucose levels of DM groups were significantly higher than the Con group at the end of the study. After the administration of resveratrol, the glucose levels were markedly decreased compared to the DM group (see Table 2). There were no significant differences between the Con group and the Res group.

\section{Bcl-2, Bax and Caspase-3 protein expression}

Western blot results show that the expression of Bcl-2 in the DM group was significantly lower than the Con group. However, resveratrol was shown to increased Bcl-2 expression (Fig. 2). Compared to the Con group, the expression of hippocampal Bax (Fig. 3) and Caspase-3 (Fig. 4) was increased in the DM group. However, hippocampal Bax and Caspase-3 protein expression was significantly decreased after 4 weeks of resveratrol administration. These results indicate that diabetes mellitus inhibits Bcl-2 expression and enhances hippocampal Bax and Caspase-3 expression. Furthermore, resveratrol treatment enhances the expression of Bcl-2 and inhibits Bax and Caspase-3 expression in the hippocampus.

Resveratrol upregulated the decreased expression of NMDAR1 and BDNF

Western blot results show that the expression of NMDAR1 and BDNF in the DM group was significantly lower than the Con group. However, resveratrol upregulated their expression after 4 weeks of administration (Fig. 5). These results indicate diabetes mellitus inhibits NMDAR1 and BDNF expression and resveratrol treatment enhances their expression.

\section{Discussion}

It has been shown that diabetes mellitus is accompanied by cognitive declines and associated with an increased risk of dementia [15]. In the present study, MWM results showed that there are significant differences between normal and diabetic rats, indicating 
that diabetes is related to cognitive impairment. This finding is consistent with previous research. Resveratrol is a natural phytoalexin enriched in grapes and red wine and has a variety of biological and pharmacological effects, such as cardioprotective, antioxidant, and anti-inflammatory activities. The impairment of cerebral vessels is a postulated underlying mechanism for diabetes mellitus-related cognitive declines [16]. A recent investigation has provided the first clinical evidence of an acute enhancement of cerebrovascular responsiveness following administration of resveratrol in this population, who are known to have endothelial dysfunction and subclinical cognitive dysfunction [17]. The research reported that maximum improvement was observed with the lowest dose used. This result is different from another randomized, double-blind, placebo-controlled, crossover study, which indicated that resveratrol administration resulted in dose-dependent increases in cerebral blood flow (where the 500-mg dose of resveratrol was more effective than the $250 \mathrm{mg}$ dose) [18]. This finding suggests that the most effective dose of resveratrol for improving cerebral blood flow may differ from age and the variety of disease. Although many studies have reported the close association between resveratrol and cognitive ability, the underlying mechanisms remain unclear. Therefore, we explored the effects of resveratrol on learning and memory ability in STZ-induced diabetic rats. Data reveal that resveratrol reverses the memory impairment and high glucose caused by diabetes. Resveratrol have no effect on normal SD rats, indicating the effect of resveratrol is a diabetes-specific effect. Our research suggests that resveratrol is a potential therapeutic and neuroprotective drug against cognitive impairment in STZ-induced diabetic model rats.

Apoptosis is an important physiological cell death process that regulates cellular homeostasis and developmental biology. Apoptosis is an important mechanism for hyperglycemia-induced hippocampal neuronal cell death. Several proteins are related to apoptosis, and the most important are caspase enzymes and proteins of the Bcl-2 family. Caspase enzymes are key regulators of apoptosis, and caspase- 3 is an important protein of this family, which plays a crucial role in promoting cell apoptosis [19]. The two main groups of Bcl-2 family, Bcl-2 and Bax proteins, are functionally opposed: Bcl-2 is the key cell apoptosis inhibitory protein, whereas Bax acts to promote apoptosis [20, 21]. The effect of Bax is achieved by releasing cytochrome $c$, which then activates caspase-3, which forms a heterodimer with $\mathrm{Bcl}-2$. Therefore, the relationship between resveratrol and the changes in Bax, Bcl-2, and caspase- 3 expression is intriguing. To explore the mechanisms underlying the therapeutic and neuroprotective effects of resveratrol in diabetic rats, we examined the expression of Bcl-2, Bax, and Caspase-3. Our results showed that hippocampal Bcl-2 was decreased, while Bax and Caspase-3 were increased in diabetic rats. However, resveratrol reverses the alteration of Bcl-2, Bax and Caspase-3 levels. These data indicate that resveratrol ameliorates the cognitive decline in STZ-induced diabetic rats, at least partly, through inhibiting hippocampal apoptosis via Bcl-2, Bax and Caspase-3 signaling pathways.

The N-methyl-d-aspartic acid receptor (NMDAR) is an important regulator of synaptic plasticity and is closely related to cognitive ability. NMDAR expression is downregulated in AD patients and models, inhibition or blockade of NMDAR results in cognitive decline [22, 23]. To explore the mechanism of resveratrol on cognitive ability in STZ-induced diabetic rats, we measured hippocampal NMDAR1 protein expression. The results indicate that diabetes mellitus inhibits NMDAR1 expression, while resveratrol treatment enhances their expression. This conclusion might imply that resveratrol ameliorates diabetes-induced cognitive impairment via modulating synaptic plasticity, but the mechanism of the effects of resveratrol on cognition, apoptosis and synaptic plasticity requires further investigation.

Brain Derived Neurotrophic Factor (BDNF), a member of the neurotrophin family of growth factors, is abundant in the central nervous system. Recent studies indicate that BDNF plays an important role in the regulation of hippocampal synaptic plasticity [24]. BDNF has also been reported to be an important regulator of neuronal apoptosis [25, 26]. In the present study, we found that after diabetic modeling, BDNF expression was decreased, while resveratrol treatment reversed the down-regulation. BDNF might play a crucial role in the resveratrol-induced regulation of synaptic plasticity and neuronal apoptosis. 


\section{Cellular Physiology Cell Physiol Biochem 2016;40:1670-1677 \begin{tabular}{ll|l} 
DOI: 10.1159/000453216 & $\begin{array}{l}\text { O 2016 The Author(s). Published by S. Karger AG, Basel } \\
\text { www.karger.com/cpb }\end{array}$
\end{tabular} \\ Tian et al.: Resveratrol and Diabetic Rats}

\section{Conclusions}

Resveratrol significantly ameliorates cognitive decline in STZ-induced diabetic model rats. The potential mechanism underlying the protective effect could be attributed to inhibition of hippocampal apoptosis through the Bcl-2, Bax and Caspase- 3 signaling pathways and improvement of synaptic dysfunction. BDNF may also play an indispensable role in this mechanism. Our results suggest resveratrol has a potential therapeutic and neuroprotective role against cognitive impairment in STZ-induced diabetic model rats and may be helpful for treating other neurological disorders.

\section{Acknowledgements}

This work was supported by the science and technology research projects of Tianjin Health Bureau (2013KG121).

\section{Disclosure Statement}

None.

\section{References}

1 Report of the expert committee on the diagnosis and classification of diabetes mellitus. Diabetes Care 2003;26:S5-20.

2 Feldman EL: Oxidative stress and diabetic neuropathy: a new understanding of an old problem. J Clin Invest 2003;111:431-433.

3 Baydas G, Nedzvetskii VS, Nerush PA, Kirichenko SV, Yoldas T: Altered expression of NCAM in hippocampus and cortex may underlie memory and learning deficits in rats with streptozotocin-induced diabetes mellitus. Life Sci 2003;73:1907-1916.

4 Sheen YJ, Sheu WH: Association between hypoglycemia and dementia in patients with type 2 diabetes. Diabetes Res Clin Pract 2016;116:279-287.

5 Areosa SA, Grimley EV: Effect of the treatment of Type II diabetes mellitus on the development of cognitive impairment and dementia. Cochrane Database Syst Rev DOI:10.1002/14651858.cd003804CD003804.

6 Guariguata L, Whiting DR, Hambleton I, Beagley J, Linnenkamp U, Shaw JE: Global estimates of diabetes prevalence for 2013 and projections for 2035. Diabetes Res Clin Pract 2014;103:137-149.

$7 \quad$ Nie P, Hu W, Zhang T, Yang Y, Hou B, Zou Z: Synergistic Induction of Erlotinib-Mediated Apoptosis by Resveratrol in Human Non-Small-Cell Lung Cancer Cells by Down-Regulating Survivin and Up-Regulating PUMA. Cell Physiol Biochem 2015;35:2255-2271.

8 Hung LM, Su MJ, Chen JK: Resveratrol protects myocardial ischemia-reperfusion injury through both NOdependent and NO-independent mechanisms. Free Radic Biol Med 2004;36:774-781.

9 Mokni M, Elkahoui S, Limam F, Amri M, Aouani E: Effect of resveratrol on antioxidant enzyme activities in the brain of healthy rat. Neurochem Res 2007;32:981-987.

10 Lin Y, Zhu J, Zhang X, Wang J, Xiao W, Li B, Jin L, Lian J, Zhou L, Liu J: Inhibition of Cardiomyocytes Hypertrophy by Resveratrol Is Associated with Amelioration of Endoplasmic Reticulum Stress. Cell Physiol Biochem 2016;39:780-789.

11 Yin K, Zhao L, Feng D, Ma W, Liu Y, Wang Y, Liang J, Yang F, Bi C, Chen H, Li X, Lu Y, Cai B: Resveratrol Attenuated Low Ambient Temperature-Induced Myocardial Hypertrophy via Inhibiting Cardiomyocyte Apoptosis. Cell Physiol Biochem 2015;35:2451-2462.

12 Wang R, Liu YY, Liu XY, Jia SW, Zhao J, Cui D, Wang L: Resveratrol protects neurons and the myocardium by reducing oxidative stress and ameliorating mitochondria damage in a cerebral ischemia rat model. Cell Physiol Biochem 2014;34:854-864. 


\section{Cellular Physiology Cell Physiol Biochem 2016;40:1670-1677 \begin{tabular}{ll|l} 
DOI: 10.1159/000453216 & $\begin{array}{l}\text { O 2016 The Author(s). Published by S. Karger AG, Basel } \\
\text { www.karger.com/cpb }\end{array}$
\end{tabular} \\ Tian et al.: Resveratrol and Diabetic Rats}

13 Schmatz R, Mazzanti CM, Spanevello R, Stefanello N, Gutierres J, Correa M, da Rosa MM, Rubin MA, Chitolina Schetinger MR, Morsch VM: Resveratrol prevents memory deficits and the increase in acetylcholinesterase activity in streptozotocin-induced diabetic rats. Eur J Pharmacol 2009;610:42-48.

14 Tiwari V, Chopra K: Resveratrol abrogates alcohol-induced cognitive deficits by attenuating oxidativenitrosative stress and inflammatory cascade in the adult rat brain. Neurochem Int 2013;62:861-869.

15 Zhang J, Liu Z, Li Z, Wang Y, Chen Y, Li X, Chen K, Shu N, Zhang Z: Disrupted White Matter Network and Cognitive Decline in Type 2 Diabetes Patients. J Alzheimers Dis 2016;53:185-195.

16 Glodzik L, Randall C, Rusinek H, de Leon MJ: Cerebrovascular reactivity to carbon dioxide in Alzheimer's disease. J Alzheimers Dis 2013;35:427-440.

17 Wong RH, Nealon RS, Scholey A, Howe PR: Low dose resveratrol improves cerebrovascular function in type 2 diabetes mellitus. Nutr Metab Cardiovasc Dis 2016;26:393-399.

18 Kennedy DO, Wightman EL, Reay JL, Lietz G, Okello EJ, Wilde A, Haskell CF: Effects of resveratrol on cerebral blood flow variables and cognitive performance in humans: a double-blind, placebo-controlled, crossover investigation. Am J Clin Nutr 2010;91:1590-1597.

19 Thornberry NA, Lazebnik Y: Caspases: enemies within. Science 1998;281:1312-1316.

20 Ashkenazi A, Dixit VM: Death receptors: signaling and modulation. Science 1998;281:1305-1308.

21 Kroemer G: The proto-oncogene Bcl-2 and its role in regulating apoptosis. Nat Med 1997;3:614-620.

22 Zhang J, Li Y, Xu J, Yang Z: The role of N-methyl-D-aspartate receptor in Alzheimer's disease. J Neurol Sci 2014;339:123-129.

23 Foster TC, Kyritsopoulos C, Kumar A: Central role for NMDA receptors in redox mediated impairment of synaptic function during aging and Alzheimer's disease. Behav Brain Res DOI:10.1016/j.bbr.2016.05.012.

24 Simmons DA, Rex CS, Palmer L, Pandyarajan V, Fedulov V, Gall CM, Lynch G: Up-regulating BDNF with an ampakine rescues synaptic plasticity and memory in Huntington's disease knockin mice. Proc Natl Acad Sci U S A 2009;106:4906-4911.

25 Hang P, Sun C, Guo J, Zhao J, Du Z: BDNF-mediates Down-regulation of MicroRNA-195 Inhibits Ischemic Cardiac Apoptosis in Rats. Int J Biol Sci 2016;12:979-989.

26 Guo D, Hou X, Zhang H, Sun W, Zhu L, Liang J, Jiang X: More expressions of BDNF and TrkB in multiple hepatocellular carcinoma and anti-BDNF or K252a induced apoptosis, supressed invasion of HepG2 and HCCLM3 cells. J Exp Clin Cancer Res 2011;30:97. 